\title{
High usage of medial unicompartmental knee arthroplasty negatively influences total knee arthroplasty revision rate
}

\author{
Antonio Klasan ${ }^{1,2,3} \cdot$ Mei Lin Tay ${ }^{4} \cdot$ Chris Frampton $^{5} \cdot$ Simon William Young Fi, $^{3,4}$
}

Received: 19 May 2021 / Accepted: 21 June 2021 / Published online: 30 June 2021

(c) The Author(s) 2021

\begin{abstract}
Purpose Surgeons with higher medial unicompartmental knee arthroplasty (UKA) usage have lower UKA revision rates. However, an increase in UKA usage may cause a decrease of total knee arthroplasty (TKA) usage. The purpose of this study was to investigate the influence of UKA usage on revision rates and patient-reported outcomes (PROMs) of UKA, TKA, and combined UKA + TKA results.

Methods Using the New Zealand Registry Database, surgeons were divided into six groups based on their medial UKA usage: $<1 \%, 1-5 \%, 5-10 \%, 10-20 \%, 20-30 \%$ and $>30 \%$. A comparison of UKA, TKA and UKA + TKA revision rates and PROMs using the Oxford Knee Score (OKS) was performed.

Results A total of 91,895 knee arthroplasties were identified, of which 8,271 were UKA (9.0\%). Surgeons with higher UKA usage had lower UKA revision rates, but higher TKA revision rates. The lowest TKA and combined UKA + TKA revision rates were observed for surgeons performing 1-5\% UKA, compared to the highest TKA and UKA+TKA revision rates which were seen for surgeons using $>30 \%$ UKA $(p<0.001$ TKA; $p<0.001$ UKA + TKA). No clinically important differences in UKA + TKA OKS scores were seen between UKA usage groups at 6 months, 5 years, or 10 years.

Conclusion Surgeons with higher medial UKA usage have lower UKA revision rates; however, this comes at the cost of a higher combined UKA + TKA revision rate that is proportionate to the UKA usage. There was no difference in TKA+UKA OKS scores between UKA usage groups. A small increase in TKA revision rate was observed for high-volume UKA users (>30\%), when compared to other UKA usage clusters. A significant decrease in UKA revision rate observed in high-volume UKA surgeons offsets the slight increase in TKA revision rate, suggesting that UKA should be performed by specialist UKA surgeons.

Level of evidence III, Retrospective therapeutic study.
\end{abstract}

Keywords Unicompartmental knee arthroplasty $\cdot$ Total knee arthroplasty $\cdot$ Survivorship $\cdot$ Revision

Antonio Klasan

klasan.antonio@me.com

1 Department for Orthopaedics and Traumatology, Kepler University Hospital GmbH, Krankenhausstrasse 9, 4020 Linz, Austria

2 Johannes Kepler University Linz, Altenberger Strasse 69, 4040 Linz, Austria

3 North Shore Hospital, 124 Shakespeare Road, Takapuna, 0620 Auckland, New Zealand

4 University of Auckland, CBD 1010, Auckland, New Zealand

5 University of Otago, 4 Oxford Terrace, 8011 Christchurch, New Zealand

\section{Introduction}

Patients with isolated medial compartment knee osteoarthritis (OA) can be treated surgically with a joint preserving high tibial osteotomy (HTO), or arthroplasty, either a medial unicompartmental knee arthroplasty (UKA) or total knee arthroplasty (TKA) [27]. In clinical practice, TKA is by far the more commonly used procedure, followed by UKA [2, 16].

If a patient undergoing arthroplasty meets the criteria for medial UKA [8], the surgeon and the patient need to decide if the faster recovery and lower perioperative morbidity after UKA are worth the cost of a higher revision rate compared to TKA $[18,30]$. According to a 2009 eligibility study, the proportion of knee arthroplasty patients suitable for UKA 
is as high as $47.6 \%$ [29]. However, mean UKA usage worldwide remains around $10-15 \%$ of knee arthroplasty [16]. Since the main drawback of UKA is the higher revision rate, mitigation of the revision rate is a strong research focus [3, $9,19,20,22]$. Surgeons with a higher UKA usage are known to have lower UKA revision rates [9]. This has led to recommendations such as a 'minimum' surgeon UKA usage of $20 \%$ and an 'optimum' usage of $40-60 \%$ to minimize the risk of revision [19]. This increase in usage is supported by studies reporting similar clinical outcomes when using 'extended' UKA indications [8], accepting higher grade patellofemoral chondral change [13], or ignoring BMI [21].

However, as most surgeons have limited scope to control the casemix within their practice, to expand their UKA usage, they will need to proportionally reduce their usage of TKA. The impact of this decrease in TKA usage on TKA revision rate, and their overall combined UKA + TKA revision rate, remains unclear. As the 'criteria' for UKA tend to include higher functioning patients with preserved range of motion and minimal deformity who also do well following TKA, it is possible that maximizing UKA usage may adversely affect surgeons' TKA results. Additionally, the 'optimum' UKA usage percentage to maximize overall patient-reported outcome measures (PROMS) remains unknown.

The aim of this study was to investigate how surgeon UKA usage influences the revision rate and PROMs of UKA, TKA, and combined UKA + TKA results.

It was hypothesized that higher UKA usage will decrease UKA revision rate. Based on the gap in the data on the influence of UKA usage on TKA and combined UKA + TKA results, a hypothesis was not possible for this research question.

\section{Methods}

Data from the New Zealand National Joint Registry (NZJR) [24] for TKA and UKA during the time period January 2000 and December 2018 was analyzed. Included were patients undergoing any knee arthroplasty for a diagnosis of osteoarthritis, performed by surgeons with greater than 100 knee arthroplasty procedures recorded on the registry. This threshold was chosen to exclude knee arthroplasty surgeons who had not yet formed a practice pattern in regards to UKA usage. Lateral unicompartmental knee arthroplasties as well as patellofemoral knee arthroplasties were excluded, since these numbers procedures are rare [15, 28].

The cases were stratified by surgeon, by procedure and by year. Each surgeon's UKA usage was calculated as the percentage of UKA from the combined number of UKA and TKA. Based on UKA usage, surgeons were then divided into six groups of UKA usage percentage: $<1.00 \% ; 1.01-5.00 \%$;
5.01-10.00\%; 10.01-20.00\%; 20.01-30.00\%; > 30.01\%. Due to a significantly higher revision rate for both UKA and TKA in patients under the age of 55 [6], a sub-analysis excluding patients under 55 at time of primary arthroplasty was also performed.

Outcome measures were all-cause revision rate of UKA, TKA, and the combined UKA + TKA revision rate per UKA usage group. Revision is defined in the NZJR as an open procedure where any component is removed, manipulated, exchanged, or implanted. Additionally, PROMS by UKA usage cluster using the Oxford Knee Score (OKS) were analyzed. The New Zealand Joint Registry collects PROMS routinely using the OKS. The Registry captures OKS after UKA and TKA at 6 months, 5 years and 10 years postoperatively, aiming for a $20 \%$ capture.

The New Zealand Joint Registry is funded from contributions from surgeons, Accident Compensation Corporation (ACC), the New Zealand government, and Southern Cross Hospital. It has an ongoing ethical approval obtained from Canterbury District Health Board in 1998. A separate ethics board approval was not necessary for the present study.

\section{Statistical analysis}

Continuous data were presented with mean $( \pm$ standard deviation) and compared with the independent $t$ test. A two-step cluster analysis was performed to determine the UKA usage clusters. With the distribution of UKA percentages, and with one cluster set to a minimum of $20.00 \%$ UKA [19], the hierarchical cluster analysis determined six clusters. Then, the $K$-means cluster analysis was used to determine the final UKA usage clusters, with the final numbers rounded to the closest 0.00 decimals. The revision rates are reported using rate/100 component years to accommodate for the different observed periods [25]. The survivorship analyses were performed using the Kaplan-Meier analyses with revision as the event. Patients were censored either at the date of death or at the end of the follow-up period, if the event has not occurred. Comparison between the clusters was performed using the Log rank (Mantel Cox) test. Due to a difference in patient demographics between the clusters, age and gender were entered into the Cox regression analysis to generate adjusted hazard ratios for cluster comparisons. Patient-reported outcomes were compared using ANOVA, and if significant effects were identified pairwise comparisons were undertaken using independent samples $t$ test. Due to the data availability as determined by the registry database size, a formal power analysis was not performed. Statistical significance was set at $p<0.05$. SPSS 24.0 (IBM, Armonk, NY, US) was used for statistical analyses. 


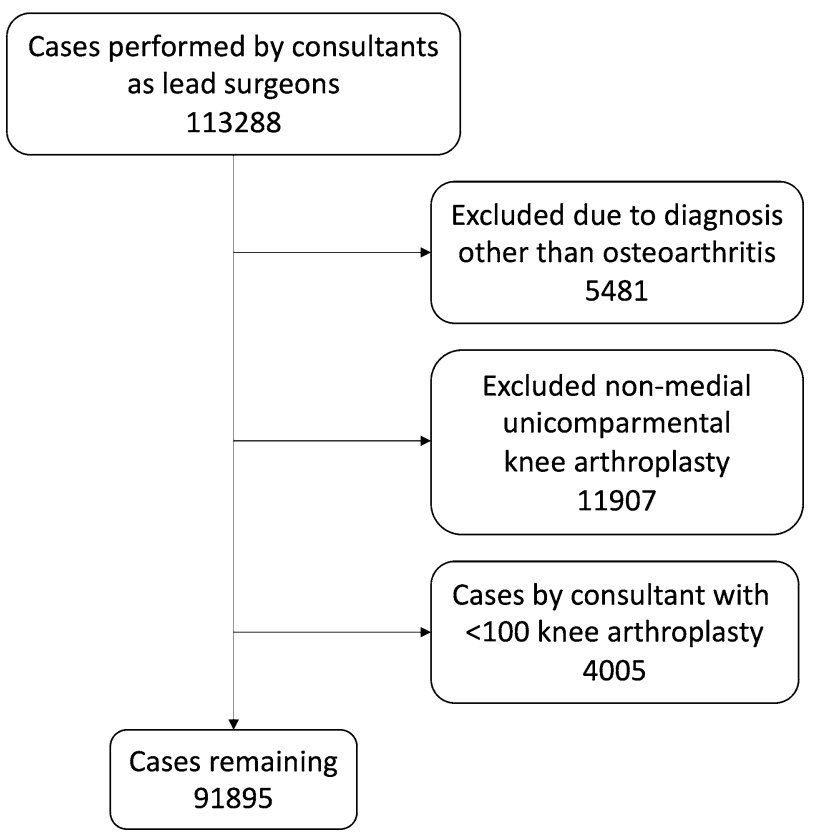

Fig. 1 Flowchart of patient inclusion

\section{Results}

In the study period, after application of inclusion and exclusion criteria, 216 consultants performed 91,895 knee arthroplasty procedures (Fig. 1). UKA was used in $9.0 \%$ of cases, 8271 procedures. An 'average' consultant performed 55 UKA and 425 TKA during a median of 14 years [3-18 years] of activity in the registry; therefore, averaging 4 UKA and 31 TKA per year. Casemix did differ between surgeon UKA usage groups, with higher UKA percentage surgeons operating on more males and younger patients $(p<0.001$, Table 1.)

The lowest overall revision rate for TKA was observed in the $1-5 \%$ UKA usage group ( 0.40 per 100 component years (cy), 95\% CI 0.64-0.44) and the highest in clusters 20-30\% UKA and <30\% UKA (both 0.59 per $100 \mathrm{cy}, 95 \%$ CI 0.51-0.67, Table 2). The lowest revision rate for UKA was observed in the $>30 \%$ UKA, of 1.0 per 100 cy $(95 \% \mathrm{CI}$ 0.86-1.14). Overall, the lowest combined UKA + TKA revision rate was seen in surgeons with a UKA usage of 1-5\% (0.44 per $100 \mathrm{cy}, 95 \%$ CI $0.4-0.48)$.

Similar findings were observed when analyzing using Kaplan-Meier survivorship. The highest UKA survivorship was observed in $>30 \%$ UKA and the lowest in $<1 \%$ UKA, Fig. 2. The higher survivorship observed in surgeons with UKA usage $>30 \%$ was statistically significant compared to all clusters except UKA usage 10-20\% (Table 3).

When only TKA outcomes are analyzed, the lowest TKA revision rate was observed in the 1-5\% UKA usage cluster, Fig. 3. Surgeons with the highest UKA usage observed a significant decrease in TKA survivorship, when compared
Table 1 Age and gender distribution between the clusters

\begin{tabular}{|c|c|c|c|c|c|c|c|c|c|}
\hline \multirow{2}{*}{$\begin{array}{l}\text { Cluster } \\
\text { UKA } \\
\text { Usage }\end{array}$} & \multirow{2}{*}{$\begin{array}{l}\text { Number } \\
\text { of surgeons }\end{array}$} & \multicolumn{2}{|l|}{ Gender } & \multirow[t]{2}{*}{ Total } & \multicolumn{4}{|c|}{ Age group } & \multirow[t]{2}{*}{ Total } \\
\hline & & $\mathrm{F}$ & M & & $<55$ & $55-64$ & $65-74$ & $\geq 75$ & \\
\hline \multicolumn{10}{|l|}{$0-1 \%$} \\
\hline$n$ & 85 & 15,063 & 14,508 & 29,571 & 2146 & 8090 & 11,680 & 7655 & 29,571 \\
\hline$\%$ & & 50.9 & 49.1 & & 7.3 & 27.4 & 39.5 & 25.9 & \\
\hline \multicolumn{10}{|l|}{$1-5 \%$} \\
\hline$n$ & 35 & 8615 & 8165 & 16,780 & 1181 & 5009 & 6516 & 4074 & 16,780 \\
\hline$\%$ & & 51.3 & 48.7 & & 7.0 & 29.9 & 38.8 & 24.3 & \\
\hline \multicolumn{10}{|l|}{$5-10 \%$} \\
\hline$n$ & 28 & 6614 & 6402 & 13,016 & 1165 & 3746 & 4910 & 3195 & 13,016 \\
\hline$\%$ & & 50.8 & 49.2 & & 9.0 & 28.8 & 37.7 & 24.5 & \\
\hline \multicolumn{10}{|l|}{$10-20 \%$} \\
\hline$n$ & 40 & 9239 & 9214 & 18,453 & 1640 & 5289 & 6887 & 4637 & 18,453 \\
\hline$\%$ & & 50.1 & 49.9 & & 8.9 & 28.7 & 37.3 & 25.1 & \\
\hline \multicolumn{10}{|l|}{$20-30 \%$} \\
\hline$n$ & 13 & 3225 & 3525 & 6750 & 558 & 1991 & 2502 & 1699 & 6750 \\
\hline$\%$ & & 47.8 & 52.2 & & 8.3 & 29.5 & 37.1 & 25.2 & \\
\hline \multicolumn{10}{|l|}{$>30 \%$} \\
\hline$n$ & 15 & 3456 & 3869 & 7325 & 658 & 2219 & 2747 & 1701 & 7325 \\
\hline$\%$ & & 47.2 & 52.8 & & 9.0 & 30.3 & 37.5 & 23.2 & \\
\hline \multicolumn{10}{|l|}{ Total } \\
\hline$n$ & 216 & 46,212 & 45,683 & 91,895 & 7348 & 26,344 & 35,242 & 22,961 & 91,895 \\
\hline$\%$ & & 50.3 & 49.7 & & 8.0 & 28.7 & 38.4 & 25.0 & \\
\hline
\end{tabular}


Table 2 Revision rates/100component-years per cluster

\begin{tabular}{|c|c|c|c|c|c|c|}
\hline $\begin{array}{l}\text { Cluster } \\
\text { UKA } \\
\text { usage }\end{array}$ & Procedures & Component years & Revised & $\begin{array}{l}\text { Rate/100- } \\
\text { component- } \\
\text { years }\end{array}$ & Lower $95 \%$ CI & Upper $95 \%$ CI \\
\hline \multicolumn{7}{|l|}{$0-1 \%$} \\
\hline TKA & 29,528 & $190,829.1$ & 917 & 0.48 & 0.45 & 0.51 \\
\hline UKA & 43 & 280.1 & 10 & 3.57 & 1.71 & 6.56 \\
\hline Total & 29,571 & $191,109.3$ & 927 & 0.49 & 0.45 & 0.52 \\
\hline \multicolumn{7}{|l|}{$1-5 \%$} \\
\hline TKA & 16,438 & $100,829.5$ & 406 & 0.40 & 0.36 & 0.44 \\
\hline UKA & 342 & 2417.8 & 51 & 2.11 & 1.55 & 2.75 \\
\hline Total & 16,780 & $103,247.3$ & 457 & 0.44 & 0.40 & 0.48 \\
\hline \multicolumn{7}{|l|}{$5-10 \%$} \\
\hline TKA & 12,118 & $82,421.1$ & 404 & 0.49 & 0.44 & 0.54 \\
\hline UKA & 898 & 7354.5 & 107 & 1.45 & 1.19 & 1.75 \\
\hline Total & 13,016 & $89,775.6$ & 511 & 0.57 & 0.52 & 0.62 \\
\hline \multicolumn{7}{|l|}{$10-20 \%$} \\
\hline TKA & 15,895 & $107,448.0$ & 493 & 0.46 & 0.42 & 0.50 \\
\hline UKA & 2558 & $17,277.5$ & 210 & 1.22 & 1.05 & 1.39 \\
\hline Total & 18,453 & $124,725.6$ & 703 & 0.56 & 0.52 & 0.61 \\
\hline \multicolumn{7}{|l|}{$20-30 \%$} \\
\hline TKA & 5199 & $35,375.4$ & 207 & 0.59 & 0.51 & 0.67 \\
\hline UKA & 1551 & 9919.5 & 133 & 1.34 & 1.12 & 1.58 \\
\hline Total & 6750 & $45,294.9$ & 340 & 0.75 & 0.67 & 0.83 \\
\hline \multicolumn{7}{|l|}{$>30 \%$} \\
\hline TKA & 4446 & $32,859.9$ & 193 & 0.59 & 0.51 & 0.67 \\
\hline UKA & 2879 & $19,459.4$ & 194 & 1.00 & 0.86 & 1.14 \\
\hline Total & 7325 & $52,319.3$ & 387 & 0.74 & 0.67 & 0.82 \\
\hline \multicolumn{7}{|l|}{ Total } \\
\hline TKA & 83,624 & $549,763.1$ & 2620 & 0.48 & 0.46 & 0.50 \\
\hline UKA & 8271 & $56,708.8$ & 705 & 1.24 & 1.15 & 1.34 \\
\hline Total & 91,895 & $606,471.9$ & 3325 & 0.55 & 0.53 & 0.57 \\
\hline
\end{tabular}

Fig. 2 Kaplan-Meier survivorship curve of only UKA survivorship, for each of the six UKA clusters, based on UKA usage

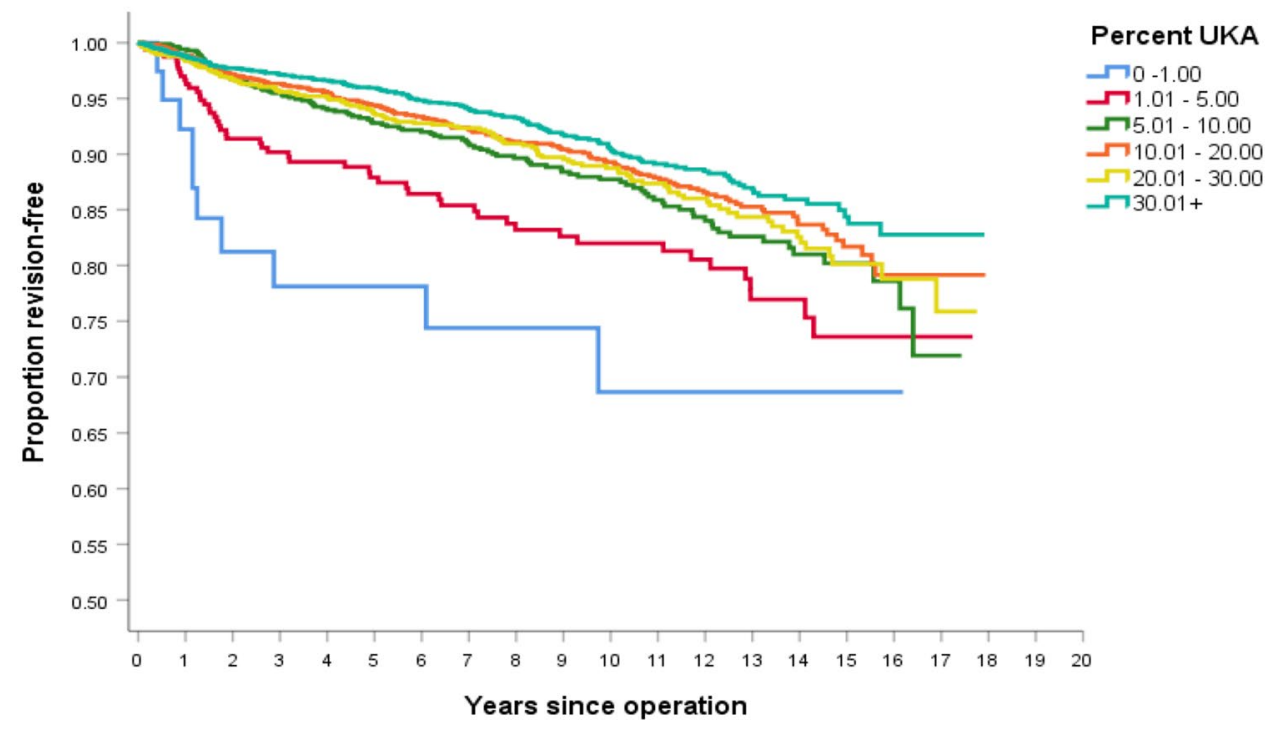


Table 3 Log rank (MantelCox) comparison of UKA survivorship between the clusters. with $p$ values reported

\begin{tabular}{lllllll}
\hline Cluster & $0-1 \%$ & $1-5 \%$ & $5-10 \%$ & $10-20 \%$ & $20-30 \%$ & $>30 \%$ \\
\hline $0-1 \%$ & & 0.140 & 0.004 & 0.001 & 0.002 & 0.000 \\
$1-5 \%$ & 0.140 & & 0.029 & 0.000 & 0.005 & 0.000 \\
$5-10 \%$ & 0.004 & 0.029 & & 0.156 & 0.503 & 0.003 \\
$10-20 \%$ & 0.001 & 0.000 & 0.156 & & 0.436 & 0.056 \\
$20-30 \%$ & 0.002 & 0.005 & 0.503 & 0.436 & & 0.014 \\
$>30 \%$ & 0.000 & 0.000 & 0.003 & 0.056 & 0.014 & \\
\hline
\end{tabular}

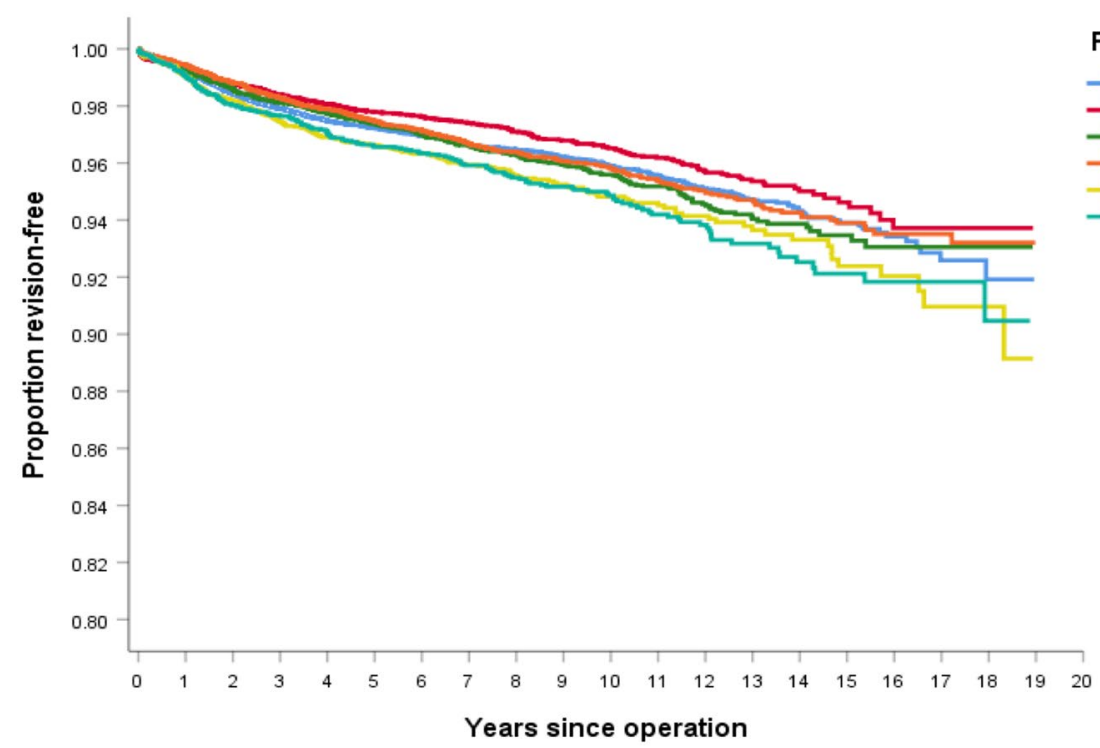

Percent UKA

Fig. 3 Kaplan-Meier survivorship curve of only TKA survivorship, for each of the six UKA clusters, based on UKA usage

Table 4 Log rank (MantelCox) comparison of TKA survivorship between the clusters, with $p$ values reported

\begin{tabular}{lllllll}
\hline Cluster & $0-1 \%$ & $1-5 \%$ & $5-10 \%$ & $10-20 \%$ & $20-30 \%$ & $>30 \%$ \\
\hline $0-1 \%$ & & 0.002 & 0.610 & 0.498 & 0.007 & 0.004 \\
$1-5 \%$ & 0.002 & & 0.002 & 0.029 & 0.000 & 0.000 \\
$5-10 \%$ & 0.610 & 0.002 & & 0.334 & 0.038 & 0.026 \\
$10-20 \%$ & 0.498 & 0.029 & 0.334 & & 0.003 & 0.002 \\
$20-30 \%$ & 0.007 & 0.000 & 0.038 & 0.003 & & 0.858 \\
$>30 \%$ & 0.004 & 0.000 & 0.026 & 0.002 & 0.858 & \\
\hline
\end{tabular}

to lower volume UKA users, Table 4. When controlling for age and gender, and excluding patients $<55$ undergoing a TKA, the lowest hazard ratio for TKA revision remained the 1-5\% UKA usage cluster, Table 5.

With increased usage of UKA, overall combined UKA + TKA survivorship decreases (Fig. 4.) There was no statistically significant difference in survivorship when compared to the cluster closest on the curve (Table 6).

When analyzing PROMS, Table 7, the overall mean OKS was higher at 6 months $(p<0.001)$ in surgeons with UKA usage $>30 \%$ than in all other groups, although the absolute difference was not clinically relevant, ranging from 0.7 to 1.6 OKS points without a clear trend between groups. At 5 years
Table 5 Cox regression analysis of TKA revision rates between clusters, controlled for age and gender, excluding patients $<55$

\begin{tabular}{lrrll}
\hline Cluster & \multicolumn{1}{c}{ Wald } & $p$ value & Hazard ratio & 95\% CI for hazard ratio \\
\hline $0-1 \%$ & 5.454 & 0.020 & 0.817 & $0.690-0.998$ \\
$1-5 \%$ & 15.366 & $<0.001$ & 0.690 & $0.573-0.830$ \\
$5-10 \%$ & 2.646 & 0.104 & 0.856 & $0.711-1.032$ \\
$10-20 \%$ & 7.701 & 0.006 & 0.772 & $0.644-0.927$ \\
$20-30 \%$ & 0.010 & 0.922 & 0.989 & $0.798-1.226$ \\
\hline
\end{tabular}

Cluster were tested against the cluster $>30 \%$

and 10 years, there was little to no difference in overall mean OKS between UKA usage groups, Table 7. 
Fig. 4 Kaplan-Meier survivorship curve of UKA + TKA survivorship, for each of the six UKA clusters, based on UKA usage

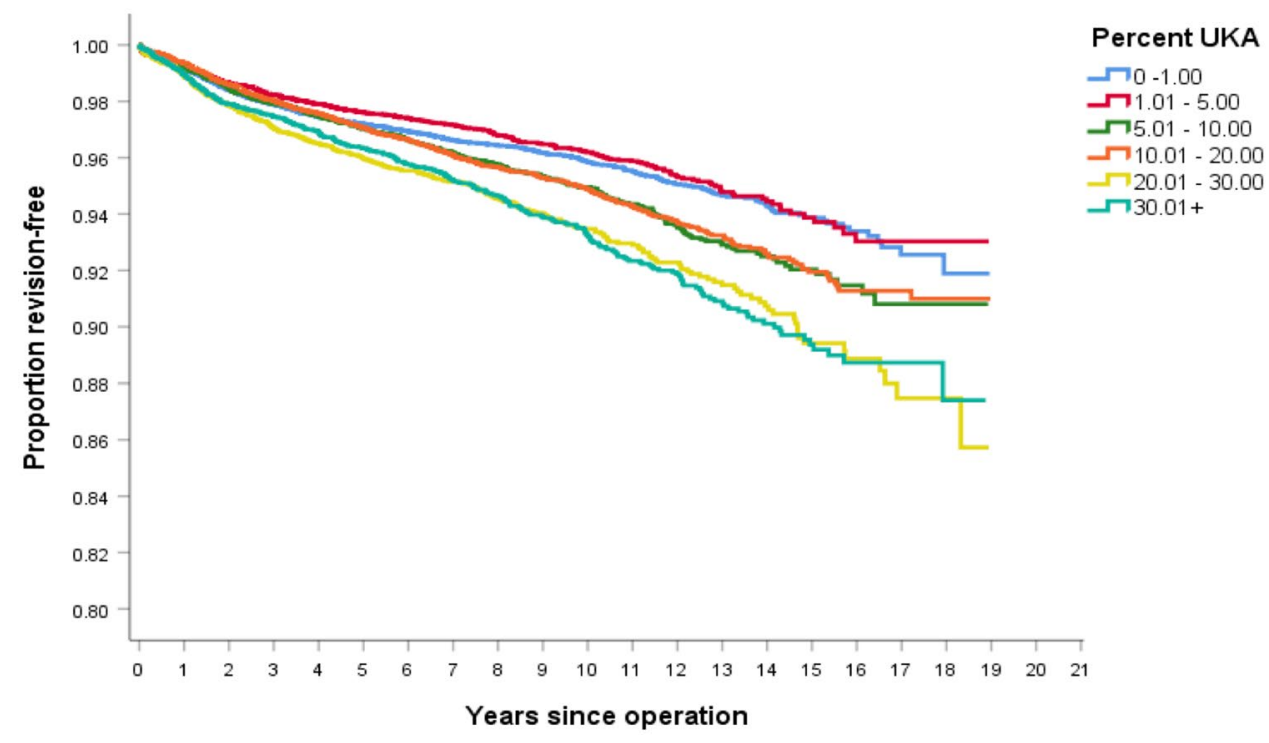

\begin{tabular}{lllllll}
\hline Cluster & $<1 \%$ & $1-5 \%$ & $5-10 \%$ & $10-20 \%$ & $20-30 \%$ & $>30 \%$ \\
\hline$<1 \%$ & & 0.077 & 0.002 & 0.002 & 0.000 & 0.000 \\
$1-5 \%$ & 0.077 & & 0.000 & 0.000 & 0.000 & 0.000 \\
$5-10 \%$ & 0.002 & 0.000 & & 0.851 & 0.000 & 0.000 \\
$10-20 \%$ & 0.002 & 0.000 & 0.851 & & 0.000 & 0.000 \\
$20-30 \%$ & 0.000 & 0.000 & 0.000 & 0.000 & & 0.912 \\
$>30 \%$ & 0.000 & 0.000 & 0.000 & 0.000 & 0.912 & \\
\hline
\end{tabular}

Table 6 Log rank (Mantel-Cox) comparison of combined UKA/ TKA survivorship between the clusters with $p$ values reported

\section{Discussion}

The most important finding of the present study is that high UKA usage is associated with a higher TKA revision rate, without an overall increase in OKS. The usage of UKA varies widely amongst knee arthroplasty surgeons, reflecting differing interpretations of the balance between advantages of UKA, such as a faster recovery and lower perioperative morbidity, versus a higher revision rate when compared to TKA $[18,30]$. Similar to previous studies, we found surgeons with higher UKA usage achieve lower UKA revision rates.

Data from the NZJR in 2006 provided one of the first reports linking higher UKA usage with lower UKA revision rates [10], and since then a number of publications have attempted to quantify the 'optimal' usage of UKA. Baker et al. analyzed the UK National Joint registry data between 2003 and 2010, categorizing surgeons and centers based on the total number of UKAs performed, ranging from $<25$ to $>200$ [3]. Improved UKA survivorship was seen in both high-volume centers and high-volume surgeons. The authors concluded that surgeons undertaking UKA should perform at least 13 UKAs per year. Similarly, Liddle et al. analyzed the UK National Joint registry using fractional polynomials, that were then fitted using locally weighted scatterplot smoothing [19]. There was a steep drop of the revision rate once $20 \%$ UKA usage was reached, with the lowest area of revision percentage observed with UKA usage of between 40 and $60 \%$. The authors concluded that, to reduce the revision risk significantly, the minimum UKA usage was $20 \%$, with 'optimum' usage is $40-60 \%$. Such studies exclusively analyze UKA outcomes. In contrast, the findings of the present study support that surgeons with high UKA usage will achieve improved UKA survivorship, their combined UKA + TKA survivorship remains lower than in surgeons with lower UKA usage percentages.

Surgeons aiming to increase their volume of UKA need to either alter their referral casemix to see more patients suitable for UKA, or broaden their indications for UKA [22]. A 2009 analysis suggested $48 \%$ of patients undergoing knee arthroplasty are potentially suitable for a UKA [29]. The findings of the present study suggest that such a high UKA percentage usage will lead to a higher overall TKA + UKA revision rate.

In evaluating combined UKA + TKA revision rates, the present study assumes that a revision from a UKA is similar to a revision from a primary TKA. Many surgeons prefer 
Table 7 Oxford knee score analysis of combined UKA/ TKA survivorship

\begin{tabular}{|c|c|c|c|c|c|c|c|}
\hline \multirow[t]{2}{*}{ Cluster } & \multirow[t]{2}{*}{$N$} & \multirow[t]{2}{*}{ Mean $( \pm S D)$} & \multicolumn{5}{|c|}{$p$ values Vs } \\
\hline & & & $1-5 \%$ & $5-10 \%$ & $10-20 \%$ & $20 \%-30 \%$ & $>30 \%$ \\
\hline \multicolumn{8}{|c|}{$\begin{array}{l}6 \text { months } \\
\text { Oxford score }\end{array}$} \\
\hline $0-1 \%$ & 7366 & $37.4( \pm 8.1)$ & $<0.001$ & $<0.001$ & $<0.001$ & 0.226 & $<0.001$ \\
\hline $1-5 \%$ & 4697 & $38.5( \pm 7.5)$ & & 0.295 & 0.169 & $<0.001$ & 0.002 \\
\hline $5-10 \%$ & 3917 & $38.3( \pm 7.7)$ & & & 0.848 & 0.001 & $<0.001$ \\
\hline $10-20 \%$ & 6270 & $38.3( \pm 7.8)$ & & & & 0.001 & $<0.001$ \\
\hline $20-30 \%$ & 2681 & $37.7( \pm 8.2)$ & & & & & $<0.001$ \\
\hline$>30$ & 3433 & $39.0( \pm 7.5)$ & & & & & \\
\hline Total & 28,364 & $38.1( \pm 7.9)$ & & & & & \\
\hline \multicolumn{8}{|c|}{$\begin{array}{l}5 \text { years } \\
\text { Oxford score }\end{array}$} \\
\hline $0-1 \%$ & 3018 & $40.5( \pm 7.7)$ & 0.027 & 0.025 & 0.189 & 0.597 & 0.019 \\
\hline $1-5 \%$ & 1745 & $41.0( \pm 7.3)$ & & 0.968 & 0.313 & 0.030 & 0.803 \\
\hline $5-10 \%$ & 1719 & $41.0( \pm 7.2)$ & & & 0.294 & 0.028 & 0.833 \\
\hline $10-20 \%$ & 2590 & $40.8( \pm 7.5)$ & & & & 0.143 & 0.222 \\
\hline $20-30 \%$ & 1004 & $40.4( \pm 7.5)$ & & & & & 0.021 \\
\hline$>30$ & 1438 & $41.1( \pm 7.3)$ & & & & & \\
\hline Total & 11,514 & $40.8( \pm 7.5)$ & & & & & \\
\hline \multicolumn{8}{|c|}{$\begin{array}{l}10 \text { years } \\
\text { Oxford score }\end{array}$} \\
\hline $0-1 \%$ & 1428 & $40.0( \pm 8.0)$ & 0.595 & 0.638 & 0.294 & 0.698 & 0.117 \\
\hline $1-5 \%$ & 894 & $40.2( \pm 8.0)$ & & 0.961 & 0.148 & 0.434 & 0.330 \\
\hline $5-10 \%$ & 859 & $40.2( \pm 8.0)$ & & & 0.168 & 0.463 & 0.312 \\
\hline $10-20 \%$ & 1318 & $39.7( \pm 8.6)$ & & & & 0.673 & 0.016 \\
\hline $20-30 \%$ & 581 & $39.9( \pm 7.9)$ & & & & & 0.104 \\
\hline$>30$ & 731 & $40.6( \pm 7.6)$ & & & & & \\
\hline Total & 5811 & $40.1( \pm 8.1)$ & & & & & \\
\hline
\end{tabular}

to use a UKA in younger patients undergoing arthroplasty, on the basis of such patients being more likely to require a revision procedure during their lifetime. The assumption is that the first revision will be more technically straightforward and have an improved outcome if it is from a primary UKA than from a primary TKA. However, a number of studies have suggested that the outcome of revision UKA is more comparable to that of a revision TKA than to a primary TKA $[17,26]$ and that revision rates of a UKA revised to a TKA are high, rendering a UKA not an intermediate procedure [12]. Furthermore, the vast majority of patients undergoing both TKA and UKA will not require a revision procedure during their lifetime, so any revision procedure can be considered an undesirable outcome, even in younger patients. The 18-year revision rate in patients $<55$ undergoing primary UKA is $40.6 \%$, compared to $17.8 \%$ for primary TKA patients in the same age group [23]. The indication for arthroplasty in a young patient should, therefore, be very carefully evaluated.

When analyzing combined UKA + TKA PROMS, an advantage in combined outcomes in the higher UKA usage (>30\%) group at 6 months, but not at 5 years or 10 years postoperatively was observed. The absolute difference of 0.7-1.4 points on the OKS is below the minimally important difference for this questionnaire, previously reported at 5 points [7]. These findings support those of previous RCTs finding similar functional outcomes for UKA and TKA [1, 4]. The most updated systematic review and meta-analysis comparing UKA and TKA patient specific outcomes and revision found no difference in pain, with functional PROMS higher for UKA than for TKA in both non-trial groups [30]. However, some TKA patients in non-trial groups may not be suitable for UKA, due to a more extensive disease, greater deformity or a significantly reduced range of motion. There may also be a difference in functional outcome in high demand patients that is not identified when using PROMS such as OKS due to the ceiling effect [14].

\section{Limitations}

There are a number of limitations in the present study. Higher usage UKA surgeons might have differing referral patterns and subsequent casemix, receiving tertiary referrals for more patients meeting UKA criteria, although there 
is also evidence against this [11]. Evidence for this is seen in the higher percentage of younger, male patients in the high UKA usage groups in the present study. However, the difference in survivorship between usage groups remained when controlling for age and gender. Furthermore, absolute differences in OKS remained small, despite the presumably higher functioning patients seen by this group. The aimed capture rate of $20 \%$ may not represent the cohort adequately. However, a large number of patients that have not been captured should have a significantly higher or lower OKS to significantly alter the scores. Furthermore, the number of patients at 10-year follow-up is lower than for shorter followups; over time there might be a difference with increased power. Second, the NZJR lacks pre-operative OKS scores and X-rays to accurately classify patients. Therefore, the specific indications of each surgeon for UKA, TKA, or other management options, such as osteotomy or non-operative therapies, remain unknown. However, by analyzing outcomes for a large number of knee surgeons across a broad time period, this study provides an overall picture of patients undergoing knee arthroplasty in New Zealand. Third, the surgeon UKA usage was grouped as a percentage of overall arthroplasty volume, which does not account for changing usage patterns during careers or an annual volume. Some studies report improved UKA outcomes with a higher absolute number of UKAs performed per year [5, 9], rather than a percentage UKA + TKA, which were not controlled in this study. However, surgeons perform more UKA cannot easily change the number of knee arthroplasty patients in their practice, rather they adjust their clinical UKA 'threshold' which is better represented by a percentage UKA usage than a volume per year. Finally, other important outcomes that may differ between UKA and TKA were not captured by this study, such as length of hospital stay, range of motion, and perioperative complications. However, revision rate and patient-reported outcomes such as the OKS remain important considerations in the decision between UKA and TKA for surgeons.

\section{Conclusions}

Surgeons with higher medial UKA usage have lower UKA revision rates; however, this comes at the cost of a higher combined UKA + TKA revision rate that is proportionate to the UKA usage. There was no difference in TKA+ UKA OKS scores between UKA usage groups. A small increase in TKA revision rate was observed for high-volume UKA users ( $>30 \%$ ), when compared to other UKA usage clusters.

A significant decrease in UKA revision rate observed in high-volume UKA surgeons offsets the slight increase in TKA revision rate, suggesting that UKA should be performed by specialist UKA surgeons.
Author contributions AK and SWY devised the study. CF did the analysis. AK and MLT wrote the first draft, AK and SWY edited it. All authors have given approval for the final version of the manuscript. All authors agree to be accountable for all aspects of the work in ensuring that questions related to the accuracy or integrity of any part of the work are appropriately investigated and resolved.

Funding Open access funding provided by Johannes Kepler University Linz. This study received no funding. The New Zealand Joint Registry is funded from contributions from surgeons, Accident Compensation Corporation, the New Zealand government, and Southern Cross Hospital.

\section{Declarations}

Conflict of interest $\mathrm{AK}$ is an editorial board member of Arch Orth Traum Surg and associate editor in BMC Musculoskelet Disord. SWY has received research support from Stryker and has been paid for presentations by Smith\&Nephew.

Ethical approval It has an ongoing ethical approval obtained from Canterbury District Health Board in 1998. A separate ethics board approval was not necessary for the present study.

Open Access This article is licensed under a Creative Commons Attribution 4.0 International License, which permits use, sharing, adaptation, distribution and reproduction in any medium or format, as long as you give appropriate credit to the original author(s) and the source, provide a link to the Creative Commons licence, and indicate if changes were made. The images or other third party material in this article are included in the article's Creative Commons licence, unless indicated otherwise in a credit line to the material. If material is not included in the article's Creative Commons licence and your intended use is not permitted by statutory regulation or exceeds the permitted use, you will need to obtain permission directly from the copyright holder. To view a copy of this licence, visit http://creativecommons.org/licenses/by/4.0/.

\section{References}

1. Ackroyd CE, Whitehouse SL, Newman JH, Joslin CC (2002) A comparative study of the medial St Georg Sled and Kinematic total knee arthroplasties. J Bone Jt Surg Br 84-B:667-672

2. Ahmed GO, ELSweify K, Ahmed AF (2020) Usability of the AAOS Appropriate Use Criteria (AUC) for the surgical management of knee osteoarthritis in clinical practice. Knee Surg Sports Traumatol Arthrosc 28:2077-2081

3. Baker P, Jameson S, Critchley R, Reed M, Gregg P, Deehan D (2013) Center and surgeon volume influence the revision rate following unicondylar knee replacement: an analysis of 23,400 medial cemented unicondylar knee replacements. J Bone Jt Surg Am 95:702-709

4. Beard DJ, Davies LJ, Cook JA, MacLennan G, Price A et al (2019) The clinical and cost-effectiveness of total versus partial knee replacement in patients with medial compartment osteoarthritis (TOPKAT): 5-year outcomes of a randomised controlled trial. Lancet 394:746-7565

5. Bini S, Khatod M, Cafri G, Chen Y, Paxton EW (2013) Surgeon, implant, and patient variables may explain variability in early revision rates reported for unicompartmental arthroplasty. J Bone Jt Surg Am 95:2195-2202

6. Charette RS, Sloan M, DeAngelis RD, Lee G-C (2019) Higher rate of early revision following primary total knee arthroplasty 
in patients under age 55: a cautionary tale. J Arthroplasty 34:2918-2924

7. Clement ND, MacDonald D, Simpson AHRW (2014) The minimal clinically important difference in the Oxford knee score and Short Form 12 score after total knee arthroplasty. Knee Surg Sports Traumatol Arthrosc 22:1933-1939

8. Hamilton TW, Pandit HG, Jenkins C, Mellon SJ, Dodd CAF, Murray DW (2017) Evidence-based indications for mobile-bearing unicompartmental knee arthroplasty in a consecutive cohort of thousand knees. J Arthroplasty 32:1779-1785

9. Hamilton TW, Rizkalla JM, Kontochristos L, Marks BE, Mellon SJ, Dodd CAF, Pandit HG, Murray DW (2017) The interaction of caseload and usage in determining outcomes of unicompartmental knee arthroplasty: a meta-analysis. J Arthroplasty 32:3228-3237. e2

10. Hartnett N, Tregonning R, Rothwell A, Hobbs T (2006) The early failure of the oxford phase 3 unicompartmental knee arthroplasty - an audit of revisions. The New Zealand experience. Orthop Proc 88-B:318-318

11. Henkel C, Mikkelsen M, Pedersen AB, Rasmussen LE, Gromov K, Price A, Troelsen A (2019) Medial unicompartmental knee arthroplasty: increasingly uniform patient demographics despite differences in surgical volume and usage-a descriptive study of 8,501 cases from the Danish Knee Arthroplasty Registry. Acta Orthop 90:354-359

12. Hunt LP, Blom AW, Matharu GS, Kunutsor SK, Beswick AD, Wilkinson JM, Whitehouse MR (2021) Patients receiving a primary unicompartmental knee replacement have a higher risk of revision but a lower risk of mortality than predicted had they received a total knee replacement: data from the national joint registry for england, Wales, Northern Ireland, and the Isle of Man. J Arthroplasty 36:471-477.e6

13. Hutt JRB, Sur A, Sur H, Ringrose A, Rickman MS (2018) Outcomes and early revision rate after medial unicompartmental knee arthroplasty: prospective results from a non-designer single surgeon. BMC Musculoskelet Disord 19:172

14. Jenny J-Y, Diesinger Y (2012) The Oxford Knee Score: compared performance before and after knee replacement. Orthop Traumatol Surg Res 98:409-412

15. Kennedy JA, Mohammad HR, Yang I, Mellon SJ, Dodd CAF, Pandit HG, Murray DW (2020) Oxford domed lateral unicompartmental knee arthroplasty. Bone Jt J 102-B:1033-1040

16. Klasan A, Parker DA, Lewis PL, Young SW (2021) Low percentage of surgeons meet the minimum recommended unicompartmental knee arthroplasty usage thresholds: analysis of 3037 Surgeons from Three National Joint Registries. Knee Surg Sports Traumatol Arthrosc. https://doi.org/10.1007/s00167-021-06437-7

17. Leta TH, Lygre SHL, Skredderstuen A, Hallan G, Gjertsen J-E, Rokne B, Furnes O (2016) Outcomes of unicompartmental knee arthroplasty after aseptic revision to total knee arthroplasty: a comparative study of 768 TKAs and 578 UKAs revised to TKAs from the Norwegian Arthroplasty Register (1994 to 2011). J Bone Jt Surg Am 98:431-440

18. Liddle AD, Judge A, Pandit H, Murray DW (2014) Adverse outcomes after total and unicompartmental knee replacement in
101,330 matched patients: a study of data from the National Joint Registry for England and Wales. Lancet 384:1437-1445

19. Liddle AD, Pandit H, Judge A, Murray DW (2015) Optimal usage of unicompartmental knee arthroplasty: a study of 41,986 cases from the National Joint Registry for England and Wales. Bone Jt J 97:1506-1511

20. Liddle AD, Pandit H, Judge A, Murray DW (2016) Effect of surgical caseload on revision rate following total and unicompartmental knee replacement. J Bone Jt Surg Am 98:1-8

21. Molloy J, Kennedy J, Jenkins C, Mellon S, Dodd C, Murray D (2019) Obesity should not be considered a contraindication to medial Oxford UKA: long-term patient-reported outcomes and implant survival in 1000 knees. Knee Surg Sports Traumatol Arthrosc 27:2259-2265

22. Murray DW, Parkinson RW (2018) Usage of unicompartmental knee arthroplasty. Bone Jt J 100-B:432-435

23. National Joint Replacement Registry AOA (2019) 2020 Hip, Knee \& Shoulder Arthroplasty Annual Report. Australian Orthopaedic Association

24. New Zealand Joint Registry NZOA (2019) Twenty year report January 1999 to December 2018

25. Pabinger C, Berghold A, Boehler N, Labek G (2013) Revision rates after knee replacement. Cumulative results from worldwide clinical studies versus joint registers. Osteoarthr Cartil 21:263-268

26. Pearse AJ, Hooper GJ, Rothwell AG, Frampton C (2012) Osteotomy and unicompartmental knee arthroplasty converted to total knee arthroplasty: data from the New Zealand Joint Registry. J Arthroplasty 27:1827-1831

27. Smith WB, Steinberg J, Scholtes S, Mcnamara IR (2017) Medial compartment knee osteoarthritis: age-stratified cost-effectiveness of total knee arthroplasty, unicompartmental knee arthroplasty, and high tibial osteotomy. Knee Surg Sports Traumatol Arthrosc 25:924-933

28. Walker T, Hariri M, Eckert J, Panzram B, Reiner T, Merle C, Gotterbarm T, Moradi B (2020) Minimally invasive lateral unicompartmental knee replacement: early results from an independent center using the Oxford fixed lateral prosthesis. Knee 27:235-241

29. Willis-Owen CA, Brust K, Alsop H, Miraldo M, Cobb JP (2009) Unicondylar knee arthroplasty in the UK National Health Service: an analysis of candidacy, outcome and cost efficacy. Knee $16: 473-478$

30. Wilson HA, Middleton R, Abram SGF, Smith S, Alvand A, Jackson WF, Bottomley N, Hopewell S, Price AJ (2019) Patient relevant outcomes of unicompartmental versus total knee replacement: systematic review and meta-analysis. BMJ 364:1352. https:// www.bmj.com/content/364/bmj.1352.abstract

Publisher's Note Springer Nature remains neutral with regard to jurisdictional claims in published maps and institutional affiliations. 